\title{
Diallel analysis of quantitative characteristics in ornamental peppers
}

\author{
R.A.G. Marcelino and A.S. Albuquerque \\ Campus Sete Lagoas, Universidade Federal de São João del Rei, Sete \\ Lagoas, MG, Brasil
}

The authors contribuite equally to this study.

Corresponding author: A.S. Albuquerque

E-mail: alejandra@ufsj.edu.br

Genet. Mol. Res. 18 (3): gmr18415

Received June 28, 2019

Accepted July 11, 2019

Published August 14, 2019

DOI http://dx.doi.org/10.4238/gmr18415

\begin{abstract}
The commerce of potted ornamental plants has grown in Brazil, and ornamental pepper stands out due to a profusion of colors and forms, as well as due to its easy cultivation. However, few studies have been carried out with ornamental pepper for genetic improvement of fruit production, plant size and precocity. Thus, in order to conduct a genetic improvement program, we evaluated the general and specific combining abilities, as well as reciprocal effects in a complete diallel with eight progenitors and 56 ornamental pepper hybrids. Thirteen features related to plant and fruits were evaluated. Significant variation was observed among parents and ornamental pepper hybrids by Scott-Knott criterion at $1 \%$ probability. There was predominance of additive effects in control of all characteristics except for fruit mass, since the ratio between quadratic components of the general combining ability and the specific combining ability was close to or greater than one. Reciprocal effects were highly significant for all traits, except for days to flowering and fruiting. The predominance of overall combining ability for most features suggests that simple genetic improvement methods, such as the pedigree method, can be
\end{abstract}


successfully used. Based on good classification of UFSJ 1 and UFSJ 6 progenitors in relation to specific combining abilities, favorable reciprocal effects for at least three characteristics simultaneously and at least one of the parents with good general combining ability, UFSJ 4 X UFSJ 1 and UFSJ 6 X UFSJ 1 hybrids were recommended for ornamental cultivation purposes.

Key words: Capsicum annuum; Ideotype; Combining ability; Reciprocal effect

\section{INTRODUCTION}

The use of autochthonous ornamental plants in the production chain aims to supply competitive market products with low environmental impact (Heiden et al., 2006). The various species of ornamental peppers originating in tropical America are inserted in this context (Reifschneider, 2000). Among potted ornamental plants, peppers have been highlighted due to their growing acceptance by the consumer market (Rêgo et al., 2009), but there are few commercial cultivars available in Brazil (Nascimento et al., 2015a). Potentially, any pepper species (Capsicum spp.) is ornamental; however, selection for this purpose is based on number of fruits, presence of erect fruits and with varied colors during the maturation process and a harmonious canopy (Santos et al., 2014).

The study of the genetic control of quantitative characteristics related to fruits and the size of ornamental peppers is of great relevance for the efficient conduction of a breeding program, using the best selection procedures and the most efficient methods for the establishment of segregating populations (Rêgo et al., 2015). In this context, diallel crosses are very efficient because they allow estimating parameters useful for the selection of parents and for understanding the genetic effects involved in determining these features (Cruz and Regazzi, 1994).

There are several methods for the analysis of diallel crosses, but the most widely used is that of Griffing (1956) because it generates information about the concentration of predominantly additive genes in its effects (general combining ability GCA) being important for the selection of parents in breeding programs. This method also generates information on genes with basically non-additive effect (dominance and epistasis), by means of the estimation of specific combining ability (SCA), which contributes to the indication of the best hybrid combination. In addition to these two parameters, important information is added in the reciprocal crosses of hybrids (REC), which indicates which genotype can be used as male or female parent in a hybrid combination according to their performance as pollen donor or recipient. The REC effect is divided into maternal effect, when it is due to the action of female nuclear genes, and extrachromosomal effect, when genes are from mitochondria and chloroplasts present in the ovule cytoplasm (Ramalho et al., 2008).

In works with Capsicum sp., several authors such as Ben-Chaim and Param (2000), Doshi and Shukla (2000), Reddy et al. (2008), Kamble et al. (2009), Rêgo et al. (2009), Schuelter et al. (2010), Gonçalves et al. (2011), Rodrigues et al. (2012), 
Medeiros et al. (2014), Nascimento et al. (2014) and Silva et al. (2017) have used the methodology of diallel analysis to increase information on several quantitative characters of interest.

This work carried out the diallel analysis of quantitative characteristics to determine the most promising crosses among eight ornamental pepper parents.

\section{MATERIAL AND METHODS}

Eight ornamental Capsicum annuum lines belonging to the germplasm bank of CSL-UFSJ (Campus Sete Lagoas, Universidade Federal de São João del Rei, Sete Lagoas, MG, Brazil) (Table 1) were crossed in complete diallel, constituting 64 treatments (eight parents and 56 hybrids) arranged in a completely randomized design, with three replicates and two plants per plot.

Table 1. Description of some qualitative traits of the eight ornamental pepper accessions (Capsicum аппиит). Plant growth habit was erect for all accessions.

\begin{tabular}{lllll}
\hline \multirow{2}{*}{ Accessions } & \multicolumn{3}{c}{ Traits } & \\
\cline { 2 - 5 } & FP & IFC & MFC & PWF \\
\hline UFSJ 1 & Erect & Yellow & Red & Intermediate \\
UFSJ 2 & Erect & Light yellow & Red & Persistent \\
UFSJ 3 & Erect & Yellow & Yellow & Intermediate \\
UFSJ 4 & Erect & Yellow & Orange & Intermediate \\
UFSJ 5 & Erect & Greenish yellow & Orange & Intermediate \\
UFSJ 6 & Erect & Light yellow & Orange & Persistent \\
UFSJ 7 & Erect & Greenish yellow & Dark red & Intermediate \\
UFSJ 8 & Pending & Light yellow & Orange and yellow & Persistent \\
\hline FP & &
\end{tabular}

$\mathrm{FP}=$ fruit position, $\mathrm{IFC}=$ fruit color at intermediate stage, $\mathrm{MFC}=$ fruit color at mature stage, $\mathrm{PWF}=$ persistence of pedicel with fruit.

To make the crosses, freshly opened flower buds of each male parent were collected to obtain pollen. The pollen obtained from each parent was stored in refrigerator in containers containing silica gel and properly identified. The flower buds of female parents were emasculated in the morning before anthesis using tweezers. In the same period, pollination was performed by placing pollen grains from each male parent on the stigmas of each emasculated flower. Each crossing was identified with a small aluminum plate tied with nylon thread in the pedicel of each pollinated flower.

Parents and their offspring were seeded in a 200-cell styrofoam tray. Seedlings were transplanted at the six-leaf stage into $900 \mathrm{~mL}$ plastic pots filled with commercial substrate (Bioplant, Brazil). Pots were kept under a mesh structure at $30 \%$ shading.

The 64 treatments were evaluated according to 13 characteristics related to fruits and plant size: canopy diameter $(\mathrm{CD})$, plant height $(\mathrm{PH})$, first bifurcation height $(\mathrm{FBH})$, stem diameter (SD), days to flowering counted from transplanting date (DTF), days to fruiting counted from transplanting date (DTFr), leaf length (LL), leaf width (LW), number of fruits per plant (FN), fruit mass (FM), fruit dry matter content (DMC), fruit length (FL) and fruit diameter (FD). All characteristics were evaluated based on 
the list of descriptors suggested by the International Plant Genetic Resources Institute (1995), and those relative to fruits were taken from 10 mature units randomly harvested on each plot. Data were submitted to analysis of variance and means were grouped by the Scott-Knott method at $1 \%$ probability.

Diallel analysis was performed by the method of Griffing (1956) using method I and model I (fixed effects). Based on this analysis, estimates of general and specific combining abilities and reciprocal effects were obtained according to the following model: Yij $=m+$ gi + gj + sij + rij + eij, in which $Y i j$ is the mean of the hybrid combination $(i \neq j)$ or parent combination $(i=j) ; m$ is the general mean; gi and gj are GCA values of ith and jth parents, respectively $(i, j=1,2, \ldots, p)$; sij is the SCA effects for crosses between ith and jth parents; rij is the reciprocal effect that measures differences made by parent $\mathrm{i}$ or $\mathrm{j}$ when used as male or female in $\mathrm{ij}$ cross; eij is the average experimental error associated with the observation of the ijk order $(\mathrm{k}=1, \ldots$, $r$ ), where $r$ is the number of replicates.

To evaluate the significant differences between GCA, SCA, and reciprocal effect, the F-test was used. The comparison between gi, sij, and rij was made by the $t$ test. All statistical analyses were performed using the Genes computer software (Cruz, 2006).

\section{RESULTS AND DISCUSSION}

Significant variations were observed for characteristics related to plant size and fruits among parents and ornamental pepper hybrids. These variations were verified through the formation of several groups for variables according to Scott-Knott criterion at $1 \%$ probability. Genotypes were separated into two groups for plant height, first bifurcation height and number of fruits per plant. Three groups were formed for stem and fruit diameters and four groups were formed for canopy diameter and fruit length. Five and seven groups were formed for fruit dry matter content and leaf width, respectively. For leaf length and fruit mass, parents and hybrids formed eight groups (Table 2).

For the 13 characteristics evaluated, there was significance for the general combining ability that represents the average behavior of parents in all hybrid combinations and covers the additive genetic variance and the additive $\mathrm{x}$ additive epistatic variance, indicating the existence of favorable alleles in all parents (Griffing, 1956). In general, the predominance of these effects was observed in the control of most characteristics evaluated for which the ratio between quadratic components of the general combining ability and quadratic components of the specific combining ability were close to or greater than the unit, except for fruit mass (FM) (Table 3). These characteristics with predominantly additive effects can be improved by simple selection scheme as the pedigree method and are easily fixed in the population. 
Table 2. Means of 13 features related to plant size and fruits evaluated in eight parents and 56 hybrids of ornamental pepper in 2018.

\begin{tabular}{|c|c|c|c|c|c|c|c|c|c|c|c|c|c|}
\hline Accessions & CD & $\mathbf{P H}$ & FBH & SD & DTF & DTFr & $\mathbf{L L}$ & LW & FN & FM & DMC & FL & FD \\
\hline 1 & $115.67 \mathrm{~d}$ & $132.73 \mathrm{a}$ & $89.83 \mathrm{a}$ & $7.37 \mathrm{~b}$ & $97.33 \mathrm{a}$ & $110.67 \mathrm{a}$ & $13.77 \mathrm{~h}$ & $8.10 \mathrm{~g}$ & $12.00 \mathrm{~b}$ & $4.22 \mathrm{a}$ & $0.67 \mathrm{~b}$ & $48.39 \mathrm{a}$ & $8.88 \mathrm{c}$ \\
\hline $1 \times 2$ & $159.33 \mathrm{~b}$ & $140.67 \mathrm{a}$ & $101.75 \mathrm{a}$ & $7.92 \mathrm{~b}$ & $100.00 \mathrm{a}$ & $111.33 \mathrm{a}$ & $13.90 \mathrm{~h}$ & $8.50 \mathrm{~g}$ & $16.00 \mathrm{~b}$ & $2.93 \mathrm{~d}$ & $0.61 \mathrm{c}$ & $25.77 \mathrm{c}$ & $10.43 \mathrm{c}$ \\
\hline $1 \times 3$ & $148.48 \mathrm{c}$ & $129.00 \mathrm{a}$ & $103.09 \mathrm{a}$ & $8.11 \mathrm{~b}$ & $102.00 \mathrm{a}$ & $115.33 \mathrm{a}$ & $13.77 \mathrm{~h}$ & $8.87 \mathrm{~g}$ & $17.33 \mathrm{~b}$ & $3.03 \mathrm{c}$ & $0.52 \mathrm{~d}$ & $26.30 \mathrm{c}$ & $9.49 \mathrm{c}$ \\
\hline $1 \times 4$ & $158.48 \mathrm{~b}$ & $133.67 \mathrm{a}$ & $87.48 \mathrm{a}$ & $5.23 \mathrm{c}$ & $94.33 \mathrm{a}$ & $108.33 \mathrm{a}$ & $14.60 \mathrm{~h}$ & $8.33 \mathrm{~g}$ & $25.00 \mathrm{a}$ & $2.16 \mathrm{f}$ & $0.47 \mathrm{~d}$ & $23.07 \mathrm{c}$ & $10.23 \mathrm{c}$ \\
\hline $1 \times 5$ & $115.62 \mathrm{~d}$ & $113.67 \mathrm{~b}$ & $83.99 \mathrm{a}$ & $6.53 \mathrm{c}$ & $94.67 \mathrm{a}$ & $107.67 \mathrm{a}$ & $14.13 \mathrm{~h}$ & $7.82 \mathrm{~g}$ & $19.00 \mathrm{~b}$ & $2.65 \mathrm{~d}$ & $0.53 \mathrm{~d}$ & $25.10 \mathrm{c}$ & $9.61 \mathrm{c}$ \\
\hline $1 \times 6$ & $92.67 \mathrm{~d}$ & $97.50 \mathrm{~b}$ & $55.50 \mathrm{~b}$ & $4.78 \mathrm{c}$ & $100.33 \mathrm{a}$ & $114.33 \mathrm{a}$ & $14.57 \mathrm{~h}$ & $8.10 \mathrm{~g}$ & $26.67 \mathrm{a}$ & $2.14 \mathrm{f}$ & $0.55 \mathrm{c}$ & $13.19 \mathrm{~d}$ & $9.75 \mathrm{c}$ \\
\hline $1 \times 7$ & $114.17 \mathrm{~d}$ & $110.33 \mathrm{~b}$ & $77.48 \mathrm{a}$ & $5.33 \mathrm{c}$ & $98.33 \mathrm{a}$ & $109.00 \mathrm{a}$ & $14.57 \mathrm{~h}$ & $8.08 \mathrm{~g}$ & $23.00 \mathrm{a}$ & $3.73 \mathrm{~b}$ & $0.69 \mathrm{~b}$ & $36.51 \mathrm{~b}$ & $9.32 \mathrm{c}$ \\
\hline $1 \times 8$ & $129.58 \mathrm{c}$ & $121.50 \mathrm{~b}$ & $86.82 \mathrm{a}$ & $6.00 \mathrm{c}$ & $98.67 \mathrm{a}$ & $115.00 \mathrm{a}$ & $14.40 \mathrm{~h}$ & $8.33 \mathrm{~g}$ & $12.33 \mathrm{~b}$ & $2.66 \mathrm{~d}$ & $0.58 \mathrm{c}$ & $48.92 \mathrm{a}$ & $15.04 \mathrm{~b}$ \\
\hline $2 \times 1$ & $111.67 \mathrm{~d}$ & $98.33 \mathrm{~b}$ & $65.47 \mathrm{~b}$ & $5.36 \mathrm{c}$ & $93.67 \mathrm{a}$ & $105.00 \mathrm{a}$ & $38.63 \mathrm{~d}$ & $20.50 \mathrm{~d}$ & $12.00 \mathrm{~b}$ & $2.32 \mathrm{e}$ & $0.68 \mathrm{~b}$ & $48.00 \mathrm{a}$ & $17.76 \mathrm{a}$ \\
\hline 2 & $129.50 \mathrm{c}$ & $111.50 \mathrm{~b}$ & $72.87 \mathrm{~b}$ & $4.67 \mathrm{c}$ & $100.67 \mathrm{a}$ & $113.67 \mathrm{a}$ & $38.78 \mathrm{~d}$ & $20.93 \mathrm{~d}$ & $17.67 \mathrm{~b}$ & $2.16 \mathrm{f}$ & $0.69 \mathrm{~b}$ & $47.62 \mathrm{a}$ & $18.14 \mathrm{a}$ \\
\hline $2 \times 3$ & $151.33 \mathrm{c}$ & $95.83 \mathrm{~b}$ & $67.47 \mathrm{~b}$ & $6.91 \mathrm{~b}$ & $88.33 \mathrm{a}$ & $104.00 \mathrm{a}$ & $38.47 \mathrm{~d}$ & $21.18 \mathrm{~d}$ & $15.67 \mathrm{~b}$ & $3.89 \mathrm{a}$ & $0.79 \mathrm{a}$ & $49.82 \mathrm{a}$ & $18.45 \mathrm{a}$ \\
\hline $2 \times 4$ & $126.83 \mathrm{c}$ & $119.45 \mathrm{~b}$ & $86.83 \mathrm{a}$ & $5.72 \mathrm{c}$ & $104.00 \mathrm{a}$ & $117.67 \mathrm{a}$ & $39.43 \mathrm{~d}$ & $21.10 \mathrm{~d}$ & $18.67 \mathrm{~b}$ & $2.67 \mathrm{~d}$ & $0.57 \mathrm{c}$ & $50.68 \mathrm{a}$ & $17.64 \mathrm{a}$ \\
\hline $2 \times 5$ & $127.00 \mathrm{c}$ & $121.00 \mathrm{~b}$ & $91.13 \mathrm{a}$ & $5.65 \mathrm{c}$ & $105.00 \mathrm{a}$ & $116.33 \mathrm{a}$ & $38.62 \mathrm{~d}$ & $22.35 \mathrm{~d}$ & $15.33 \mathrm{~b}$ & $2.88 \mathrm{~d}$ & $0.65 \mathrm{~b}$ & $50.90 \mathrm{a}$ & $18.10 \mathrm{a}$ \\
\hline $2 \times 6$ & $123.13 \mathrm{c}$ & $101.53 \mathrm{~b}$ & $77.46 \mathrm{a}$ & $5.58 \mathrm{c}$ & $110.00 \mathrm{a}$ & $120.33 \mathrm{a}$ & $39.63 \mathrm{~d}$ & $22.23 \mathrm{~d}$ & $21.33 \mathrm{a}$ & $3.05 \mathrm{c}$ & $0.60 \mathrm{c}$ & $47.53 \mathrm{a}$ & $18.72 \mathrm{a}$ \\
\hline $2 \times 7$ & $152.53 \mathrm{c}$ & $112.40 \mathrm{~b}$ & $80.66 \mathrm{a}$ & $5.60 \mathrm{c}$ & $108.00 \mathrm{a}$ & $121.00 \mathrm{a}$ & $38.67 \mathrm{~d}$ & $21.68 \mathrm{~d}$ & $21.00 \mathrm{a}$ & $2.86 \mathrm{~d}$ & $0.51 \mathrm{~d}$ & $24.51 \mathrm{c}$ & $17.26 \mathrm{a}$ \\
\hline $2 \times 8$ & $125.00 \mathrm{c}$ & $90.55 \mathrm{~b}$ & $68.89 \mathrm{~b}$ & $4.97 \mathrm{c}$ & $109.33 \mathrm{a}$ & $124.33 \mathrm{a}$ & $30.80 \mathrm{f}$ & $14.27 \mathrm{f}$ & $13.33 \mathrm{~b}$ & $3.60 \mathrm{~b}$ & $0.76 \mathrm{a}$ & $26.03 \mathrm{c}$ & $13.44 \mathrm{~b}$ \\
\hline $3 \times 1$ & $145.08 \mathrm{c}$ & $115.17 \mathrm{~b}$ & $78.67 \mathrm{a}$ & $7.33 \mathrm{~b}$ & $101.67 \mathrm{a}$ & $119.67 \mathrm{a}$ & $31.23 \mathrm{f}$ & $14.50 \mathrm{f}$ & $13.00 \mathrm{~b}$ & $2.81 \mathrm{~d}$ & $0.54 \mathrm{c}$ & $25.57 \mathrm{c}$ & $14.27 \mathrm{~b}$ \\
\hline $3 \times 2$ & $183.17 \mathrm{~b}$ & $115.00 \mathrm{~b}$ & $82.67 \mathrm{a}$ & $6.17 \mathrm{c}$ & $89.00 \mathrm{a}$ & $103.00 \mathrm{a}$ & $32.17 \mathrm{f}$ & $14.83 \mathrm{f}$ & $10.33 \mathrm{~b}$ & $3.16 \mathrm{c}$ & $0.79 \mathrm{a}$ & $24.31 \mathrm{c}$ & $13.88 \mathrm{~b}$ \\
\hline 3 & $106.33 \mathrm{~d}$ & $85.83 \mathrm{~b}$ & $56.17 \mathrm{~b}$ & $5.55 \mathrm{c}$ & $93.67 \mathrm{a}$ & $108.33 \mathrm{a}$ & $31.63 \mathrm{f}$ & $13.97 \mathrm{f}$ & $15.33 \mathrm{~b}$ & $3.53 \mathrm{~b}$ & $0.75 \mathrm{a}$ & $12.61 \mathrm{~d}$ & $12.88 \mathrm{~b}$ \\
\hline $3 \times 4$ & $151.10 \mathrm{c}$ & $101.17 \mathrm{~b}$ & $74.67 \mathrm{~b}$ & $6.83 \mathrm{~b}$ & $86.33 \mathrm{a}$ & $102.33 \mathrm{a}$ & $31.77 \mathrm{f}$ & $14.10 \mathrm{f}$ & $14.67 \mathrm{~b}$ & $2.66 \mathrm{~d}$ & $0.57 \mathrm{c}$ & $24.90 \mathrm{c}$ & $12.96 \mathrm{~b}$ \\
\hline $3 \times 5$ & $105.33 \mathrm{~d}$ & $80.83 \mathrm{~b}$ & $47.50 \mathrm{~b}$ & $8.22 \mathrm{~b}$ & $100.33 \mathrm{a}$ & $118.00 \mathrm{a}$ & $31.23 \mathrm{f}$ & $14.53 \mathrm{f}$ & $20.33 \mathrm{a}$ & $2.99 \mathrm{c}$ & $0.77 \mathrm{a}$ & $24.89 \mathrm{c}$ & $15.17 \mathrm{~b}$ \\
\hline $3 \times 6$ & $133.53 \mathrm{c}$ & $100.20 \mathrm{~b}$ & $74.80 \mathrm{~b}$ & $8.43 \mathrm{~b}$ & $88.00 \mathrm{a}$ & $106.00 \mathrm{a}$ & $30.83 \mathrm{f}$ & $14.50 \mathrm{f}$ & $18.33 \mathrm{~b}$ & $3.23 \mathrm{c}$ & $0.80 \mathrm{a}$ & $23.52 \mathrm{c}$ & $13.69 \mathrm{~b}$ \\
\hline $3 \times 7$ & $135.83 \mathrm{c}$ & $129.25 \mathrm{a}$ & $82.00 \mathrm{a}$ & $6.83 \mathrm{~b}$ & $98.67 \mathrm{a}$ & $111.00 \mathrm{a}$ & $30.57 \mathrm{f}$ & $14.50 \mathrm{f}$ & $18.67 \mathrm{~b}$ & $3.35 \mathrm{~b}$ & $0.88 \mathrm{a}$ & $25.17 \mathrm{c}$ & $15.42 \mathrm{~b}$ \\
\hline $3 \times 8$ & $176.47 \mathrm{~b}$ & $134.72 \mathrm{a}$ & $95.67 \mathrm{a}$ & $7.83 \mathrm{~b}$ & $112.00 \mathrm{a}$ & $127.33 \mathrm{a}$ & $45.67 \mathrm{c}$ & $17.23 \mathrm{e}$ & $11.33 \mathrm{~b}$ & $2.94 \mathrm{~d}$ & $0.64 \mathrm{~b}$ & $23.10 \mathrm{c}$ & $14.24 \mathrm{~b}$ \\
\hline $4 \times 1$ & $106.50 \mathrm{~d}$ & $83.67 \mathrm{~b}$ & $56.00 \mathrm{~b}$ & $4.96 \mathrm{c}$ & $100.67 \mathrm{a}$ & $117.33 \mathrm{a}$ & $46.70 \mathrm{c}$ & $17.57 \mathrm{e}$ & $19.33 \mathrm{a}$ & $3.29 \mathrm{c}$ & $0.80 \mathrm{a}$ & $23.02 \mathrm{c}$ & $18.18 \mathrm{a}$ \\
\hline $4 \times 2$ & $167.17 \mathrm{~b}$ & $136.92 \mathrm{a}$ & $97.50 \mathrm{a}$ & $7.33 \mathrm{~b}$ & $95.67 \mathrm{a}$ & $113.33 \mathrm{a}$ & $46.33 c$ & $17.37 \mathrm{e}$ & $13.67 \mathrm{~b}$ & $3.73 \mathrm{~b}$ & $0.81 \mathrm{a}$ & $21.17 \mathrm{c}$ & $16.33 \mathrm{~b}$ \\
\hline $4 \times 3$ & $149.47 \mathrm{c}$ & $97.80 \mathrm{~b}$ & $69.57 \mathrm{~b}$ & $7.50 \mathrm{~b}$ & $101.67 \mathrm{a}$ & $113.67 \mathrm{a}$ & $46.07 \mathrm{c}$ & $17.13 \mathrm{e}$ & $19.67 \mathrm{a}$ & $1.95 \mathrm{~g}$ & $0.43 \mathrm{~d}$ & $24.24 \mathrm{c}$ & $15.77 \mathrm{~b}$ \\
\hline 4 & $157.50 \mathrm{~b}$ & $133.50 \mathrm{a}$ & $93.17 \mathrm{a}$ & $7.23 \mathrm{~b}$ & $96.00 \mathrm{a}$ & $107.33 \mathrm{a}$ & $47.20 \mathrm{c}$ & $18.70 \mathrm{~d}$ & $21.00 \mathrm{a}$ & $1.93 \mathrm{~g}$ & $0.37 \mathrm{e}$ & $25.77 \mathrm{c}$ & $14.87 \mathrm{~b}$ \\
\hline $4 \times 5$ & $148.52 \mathrm{c}$ & $113.44 \mathrm{~b}$ & $80.67 \mathrm{a}$ & $6.66 \mathrm{~b}$ & $91.33 \mathrm{a}$ & $108.67 \mathrm{a}$ & $46.77 \mathrm{c}$ & $18.40 \mathrm{e}$ & $16.33 \mathrm{~b}$ & $1.26 \mathrm{~h}$ & $0.31 \mathrm{e}$ & $12.60 \mathrm{~d}$ & $14.33 \mathrm{~b}$ \\
\hline $4 \times 6$ & $146.88 \mathrm{c}$ & $118.00 \mathrm{~b}$ & $83.33 \mathrm{a}$ & $7.27 \mathrm{~b}$ & $84.67 \mathrm{a}$ & $98.67 \mathrm{a}$ & $47.47 \mathrm{c}$ & $17.77 \mathrm{e}$ & $19.33 \mathrm{a}$ & $1.75 \mathrm{~g}$ & $0.46 \mathrm{~d}$ & $19.07 \mathrm{c}$ & $13.84 \mathrm{~b}$ \\
\hline $4 \times 7$ & $143.67 \mathrm{c}$ & $128.35 \mathrm{a}$ & $84.50 \mathrm{a}$ & $7.92 \mathrm{~b}$ & $95.00 \mathrm{a}$ & $107.67 \mathrm{a}$ & $45.77 \mathrm{c}$ & $17.37 \mathrm{e}$ & $16.00 \mathrm{~b}$ & $1.59 \mathrm{~g}$ & $0.32 \mathrm{e}$ & $27.20 \mathrm{c}$ & $14.13 \mathrm{~b}$ \\
\hline $4 \times 8$ & $148.67 \mathrm{c}$ & $111.67 \mathrm{~b}$ & $80.00 \mathrm{a}$ & $5.65 \mathrm{c}$ & $96.00 \mathrm{a}$ & $107.33 \mathrm{a}$ & $33.47 \mathrm{e}$ & $24.20 \mathrm{c}$ & $15.00 \mathrm{~b}$ & $2.10 \mathrm{f}$ & $0.41 \mathrm{e}$ & $24.91 \mathrm{c}$ & $17.77 \mathrm{a}$ \\
\hline $5 \times 1$ & $165.25 \mathrm{~b}$ & $107.98 \mathrm{~b}$ & $81.66 \mathrm{a}$ & $6.37 \mathrm{c}$ & $102.67 \mathrm{a}$ & $116.00 \mathrm{a}$ & $33.77 \mathrm{e}$ & $24.10 \mathrm{c}$ & $13.67 \mathrm{~b}$ & $2.32 \mathrm{e}$ & $0.35 \mathrm{e}$ & $25.95 \mathrm{c}$ & $20.82 \mathrm{a}$ \\
\hline $5 \times 2$ & $117.70 \mathrm{~d}$ & $89.67 \mathrm{~b}$ & $54.00 \mathrm{~b}$ & $4.33 \mathrm{c}$ & $99.67 \mathrm{a}$ & $114.00 \mathrm{a}$ & $33.17 \mathrm{e}$ & $25.07 \mathrm{c}$ & $11.33 \mathrm{~b}$ & $2.31 \mathrm{e}$ & $0.37 \mathrm{e}$ & $26.01 \mathrm{c}$ & $16.28 \mathrm{~b}$ \\
\hline $5 \times 3$ & $80.17 \mathrm{~d}$ & $77.00 \mathrm{~b}$ & $49.17 \mathrm{~b}$ & $5.83 \mathrm{c}$ & $96.67 \mathrm{a}$ & $107.67 \mathrm{a}$ & $34.27 \mathrm{e}$ & $24.80 \mathrm{c}$ & $20.33 \mathrm{a}$ & $2.45 \mathrm{e}$ & $0.36 \mathrm{e}$ & $22.65 \mathrm{c}$ & $21.96 \mathrm{a}$ \\
\hline $5 \times 4$ & $102.67 \mathrm{~d}$ & $80.67 \mathrm{~b}$ & $56.67 \mathrm{~b}$ & $6.23 \mathrm{c}$ & $103.00 \mathrm{a}$ & $120.00 \mathrm{a}$ & $33.93 \mathrm{e}$ & $24.67 \mathrm{c}$ & $15.00 \mathrm{~b}$ & $1.24 \mathrm{~h}$ & $0.29 \mathrm{e}$ & $26.61 \mathrm{c}$ & $20.95 \mathrm{a}$ \\
\hline 5 & $116.18 \mathrm{~d}$ & $94.30 \mathrm{~b}$ & $70.87 \mathrm{~b}$ & $5.60 \mathrm{c}$ & $85.67 \mathrm{a}$ & $102.00 \mathrm{a}$ & $35.00 \mathrm{e}$ & $25.23 \mathrm{c}$ & $15.67 \mathrm{~b}$ & $1.31 \mathrm{~h}$ & $0.31 \mathrm{e}$ & $26.69 \mathrm{c}$ & $19.37 \mathrm{a}$ \\
\hline $5 \times 6$ & $104.83 \mathrm{~d}$ & $90.10 \mathrm{~b}$ & $56.80 \mathrm{~b}$ & $6.80 \mathrm{~b}$ & $106.67 \mathrm{a}$ & $121.67 \mathrm{a}$ & $34.10 \mathrm{e}$ & $24.67 \mathrm{c}$ & $15.33 \mathrm{~b}$ & $1.65 \mathrm{~g}$ & $0.33 \mathrm{e}$ & $24.28 \mathrm{c}$ & $18.49 \mathrm{a}$ \\
\hline $5 \times 7$ & $163.17 \mathrm{~b}$ & $139.33 \mathrm{a}$ & $86.67 \mathrm{a}$ & $7.57 \mathrm{~b}$ & $104.00 \mathrm{a}$ & $115.33 \mathrm{a}$ & $34.27 \mathrm{e}$ & $24.57 \mathrm{c}$ & $15.33 \mathrm{~b}$ & $1.99 \mathrm{~g}$ & $0.31 \mathrm{e}$ & $13.97 \mathrm{~d}$ & $19.51 \mathrm{a}$ \\
\hline $5 \times 8$ & $150.97 \mathrm{c}$ & $125.92 \mathrm{a}$ & $95.50 \mathrm{a}$ & $6.00 \mathrm{c}$ & $97.67 \mathrm{a}$ & $108.33 \mathrm{a}$ & $19.27 \mathrm{~g}$ & $15.33 \mathrm{f}$ & $14.00 \mathrm{~b}$ & $2.38 \mathrm{e}$ & $0.39 \mathrm{e}$ & $21.02 \mathrm{c}$ & $13.60 \mathrm{~b}$ \\
\hline $6 \times 1$ & $134.75 \mathrm{c}$ & $103.00 \mathrm{~b}$ & $81.83 \mathrm{a}$ & $6.93 \mathrm{~b}$ & $97.00 \mathrm{a}$ & $108.33 \mathrm{a}$ & $21.20 \mathrm{~g}$ & $16.10 \mathrm{e}$ & $20.33 \mathrm{a}$ & $2.55 \mathrm{e}$ & $0.41 \mathrm{e}$ & $19.22 \mathrm{c}$ & $13.27 \mathrm{~b}$ \\
\hline $6 \times 2$ & $125.83 \mathrm{c}$ & $113.17 \mathrm{~b}$ & $78.17 \mathrm{a}$ & $4.72 \mathrm{c}$ & $91.67 \mathrm{a}$ & $109.33 \mathrm{a}$ & $20.53 \mathrm{~g}$ & $16.30 \mathrm{e}$ & $12.00 \mathrm{~b}$ & $2.30 \mathrm{e}$ & $0.39 \mathrm{e}$ & $21.03 \mathrm{c}$ & $14.09 \mathrm{~b}$ \\
\hline $6 \times 3$ & $86.00 \mathrm{~d}$ & $88.33 \mathrm{~b}$ & $54.50 \mathrm{~b}$ & $4.38 \mathrm{c}$ & $98.33 \mathrm{a}$ & $111.00 \mathrm{a}$ & $21.23 \mathrm{~g}$ & $16.50 \mathrm{e}$ & $20.67 \mathrm{a}$ & $2.22 \mathrm{f}$ & $0.35 \mathrm{e}$ & $26.87 \mathrm{c}$ & $14.25 \mathrm{~b}$ \\
\hline $6 \times 4$ & $123.42 \mathrm{c}$ & $121.30 \mathrm{~b}$ & $84.83 \mathrm{a}$ & $5.42 \mathrm{c}$ & $104.33 \mathrm{a}$ & $117.67 \mathrm{a}$ & $21.73 \mathrm{~g}$ & $16.27 \mathrm{e}$ & $13.33 \mathrm{~b}$ & $1.97 \mathrm{~g}$ & $0.33 \mathrm{e}$ & $20.90 \mathrm{c}$ & $14.34 \mathrm{~b}$ \\
\hline $6 \times 5$ & $136.30 \mathrm{c}$ & $111.40 \mathrm{~b}$ & $96.43 \mathrm{a}$ & $5.24 \mathrm{c}$ & $104.00 \mathrm{a}$ & $118.67 \mathrm{a}$ & $21.37 \mathrm{~g}$ & $16.13 \mathrm{e}$ & $19.00 \mathrm{~b}$ & $1.85 \mathrm{~g}$ & $0.30 \mathrm{e}$ & $20.22 \mathrm{c}$ & $13.08 \mathrm{~b}$ \\
\hline 6 & $158.08 \mathrm{~b}$ & $119.67 \mathrm{~b}$ & $77.00 \mathrm{a}$ & $6.33 \mathrm{c}$ & $97.67 \mathrm{a}$ & $112.33 \mathrm{a}$ & $21.90 \mathrm{~g}$ & $16.57 \mathrm{e}$ & $20.33 \mathrm{a}$ & $1.88 \mathrm{~g}$ & $0.32 \mathrm{e}$ & $21.36 \mathrm{c}$ & $14.09 \mathrm{~b}$ \\
\hline $6 \times 7$ & $190.67 \mathrm{a}$ & $147.50 \mathrm{a}$ & $109.50 \mathrm{a}$ & $8.50 \mathrm{~b}$ & $95.00 \mathrm{a}$ & $110.67 \mathrm{a}$ & $22.90 \mathrm{~g}$ & $15.53 \mathrm{f}$ & $10.67 \mathrm{~b}$ & $2.33 \mathrm{e}$ & $0.39 \mathrm{e}$ & $26.05 \mathrm{c}$ & $16.43 \mathrm{~b}$ \\
\hline $6 \times 8$ & $155.77 \mathrm{~b}$ & $107.60 \mathrm{~b}$ & $80.33 \mathrm{a}$ & $7.63 \mathrm{~b}$ & $95.33 \mathrm{a}$ & $108.00 \mathrm{a}$ & $60.30 \mathrm{~b}$ & $27.73 \mathrm{~b}$ & $18.00 \mathrm{~b}$ & $1.67 \mathrm{~g}$ & $0.35 \mathrm{e}$ & $24.97 \mathrm{c}$ & $7.23 \mathrm{c}$ \\
\hline $7 \times 1$ & $191.60 \mathrm{a}$ & $126.67 \mathrm{a}$ & $95.70 \mathrm{a}$ & $7.75 \mathrm{~b}$ & $107.67 \mathrm{a}$ & $122.33 \mathrm{a}$ & $62.10 \mathrm{~b}$ & $28.73 \mathrm{~b}$ & $15.67 \mathrm{~b}$ & $2.14 \mathrm{f}$ & $0.40 \mathrm{e}$ & $13.38 \mathrm{~d}$ & $8.22 \mathrm{c}$ \\
\hline $7 \times 2$ & $177.75 \mathrm{~b}$ & $138.07 \mathrm{a}$ & $98.00 \mathrm{a}$ & $8.35 \mathrm{~b}$ & $112.00 \mathrm{a}$ & $130.33 \mathrm{a}$ & $62.30 \mathrm{~b}$ & $28.20 \mathrm{~b}$ & $23.33 \mathrm{a}$ & $2.88 \mathrm{~d}$ & $0.47 \mathrm{~d}$ & $26.30 \mathrm{c}$ & $7.53 \mathrm{c}$ \\
\hline $7 \times 3$ & $176.60 \mathrm{~b}$ & $140.67 \mathrm{a}$ & $102.58 \mathrm{a}$ & $11.16 \mathrm{a}$ & $113.00 \mathrm{a}$ & $131.33 \mathrm{a}$ & $60.43 \mathrm{~b}$ & $27.30 \mathrm{~b}$ & $17.33 \mathrm{~b}$ & $2.07 \mathrm{f}$ & $0.38 \mathrm{e}$ & $26.16 \mathrm{c}$ & $8.30 \mathrm{c}$ \\
\hline $7 \times 4$ & $189.00 \mathrm{a}$ & $138.00 \mathrm{a}$ & $105.00 \mathrm{a}$ & $8.48 \mathrm{~b}$ & $110.00 \mathrm{a}$ & $122.33 \mathrm{a}$ & $60.50 \mathrm{~b}$ & $26.53 \mathrm{~b}$ & $15.67 \mathrm{~b}$ & $2.38 \mathrm{e}$ & $0.36 \mathrm{e}$ & $25.30 \mathrm{c}$ & $8.37 \mathrm{c}$ \\
\hline $7 \times 5$ & $149.67 \mathrm{c}$ & $117.67 \mathrm{~b}$ & $78.33 \mathrm{a}$ & $8.17 \mathrm{~b}$ & $107.67 \mathrm{a}$ & $122.33 \mathrm{a}$ & $61.47 \mathrm{~b}$ & $27.63 \mathrm{~b}$ & $15.67 \mathrm{~b}$ & $2.92 \mathrm{~d}$ & $0.50 \mathrm{~d}$ & $25.28 \mathrm{c}$ & $8.39 \mathrm{c}$ \\
\hline $7 \times 6$ & $189.00 \mathrm{a}$ & $146.67 \mathrm{a}$ & $103.00 \mathrm{a}$ & $9.90 \mathrm{a}$ & $107.00 \mathrm{a}$ & $123.33 \mathrm{a}$ & $61.67 \mathrm{~b}$ & $25.57 \mathrm{c}$ & $22.67 \mathrm{a}$ & $2.85 \mathrm{~d}$ & $0.45 \mathrm{~d}$ & $20.23 \mathrm{c}$ & $8.79 \mathrm{c}$ \\
\hline 7 & $173.87 \mathrm{~b}$ & $135.33 \mathrm{a}$ & $89.00 \mathrm{a}$ & $10.28 \mathrm{a}$ & $99.00 \mathrm{a}$ & $112.00 \mathrm{a}$ & $62.03 \mathrm{~b}$ & $27.27 \mathrm{~b}$ & $22.00 \mathrm{a}$ & $2.71 \mathrm{~d}$ & $0.44 \mathrm{~d}$ & $13.09 \mathrm{~d}$ & $8.22 \mathrm{c}$ \\
\hline $7 \times 8$ & $207.83 \mathrm{a}$ & $136.00 \mathrm{a}$ & $93.00 \mathrm{a}$ & $11.87 \mathrm{a}$ & $109.67 \mathrm{a}$ & $123.00 \mathrm{a}$ & $65.10 \mathrm{a}$ & $37.30 \mathrm{a}$ & $25.00 \mathrm{a}$ & $2.21 \mathrm{f}$ & $0.38 \mathrm{e}$ & $12.76 \mathrm{~d}$ & $11.90 \mathrm{c}$ \\
\hline $8 \times 1$ & $204.17 \mathrm{a}$ & $155.83 \mathrm{a}$ & $101.10 \mathrm{a}$ & $10.03 \mathrm{a}$ & $119.00 \mathrm{a}$ & $135.67 \mathrm{a}$ & $65.63 \mathrm{a}$ & $36.77 \mathrm{a}$ & $24.33 \mathrm{a}$ & $1.79 \mathrm{~g}$ & $0.39 \mathrm{e}$ & $12.93 \mathrm{~d}$ & $10.81 \mathrm{c}$ \\
\hline $8 \times 2$ & $183.17 \mathrm{~b}$ & $137.83 \mathrm{a}$ & $100.93 \mathrm{a}$ & $10.50 \mathrm{a}$ & $121.67 \mathrm{a}$ & $136.33 \mathrm{a}$ & $65.33 \mathrm{a}$ & $36.87 \mathrm{a}$ & $27.00 \mathrm{a}$ & $1.89 \mathrm{~g}$ & $0.32 \mathrm{e}$ & $14.35 \mathrm{~d}$ & $9.05 \mathrm{c}$ \\
\hline $8 \times 3$ & $210.83 \mathrm{a}$ & $169.83 \mathrm{a}$ & $99.00 \mathrm{a}$ & $10.58 \mathrm{a}$ & $118.00 \mathrm{a}$ & $134.33 \mathrm{a}$ & $66.07 \mathrm{a}$ & $35.27 \mathrm{a}$ & $18.00 \mathrm{~b}$ & $2.06 \mathrm{f}$ & $0.36 \mathrm{e}$ & $13.04 \mathrm{~d}$ & $9.25 \mathrm{c}$ \\
\hline $8 \times 4$ & $212.13 \mathrm{a}$ & & $97.00 \mathrm{a}$ & $12.55 \mathrm{a}$ & $117.00 \mathrm{a}$ & $132.00 \mathrm{a}$ & $68.20 \mathrm{a}$ & $37.27 \mathrm{a}$ & $25.33 \mathrm{a}$ & $1.84 \mathrm{~g}$ & $0.35 \mathrm{e}$ & $13.93 \mathrm{~d}$ & $9.76 \mathrm{c}$ \\
\hline $8 \times 5$ & $172.67 \mathrm{~b}$ & $114.63 \mathrm{~b}$ & $78.33 \mathrm{a}$ & $8.25 \mathrm{~b}$ & $95.00 \mathrm{a}$ & $110.67 \mathrm{a}$ & $66.27 \mathrm{a}$ & $37.83 \mathrm{a}$ & $28.33 \mathrm{a}$ & $2.11 \mathrm{f}$ & $0.38 \mathrm{e}$ & $14.50 \mathrm{~d}$ & $9.07 \mathrm{c}$ \\
\hline $8 \times 6$ & $217.00 \mathrm{a}$ & $167.25 \mathrm{a}$ & $104.83 \mathrm{a}$ & $12.42 \mathrm{a}$ & $109.00 \mathrm{a}$ & $123.67 \mathrm{a}$ & $66.40 \mathrm{a}$ & $34.93 \mathrm{a}$ & $30.00 \mathrm{a}$ & $1.93 \mathrm{~g}$ & $0.36 \mathrm{e}$ & $14.76 \mathrm{~d}$ & $10.96 \mathrm{c}$ \\
\hline $8 \times 7$ & $211.38 \mathrm{a}$ & $175.00 \mathrm{a}$ & $108.50 \mathrm{a}$ & $12.50 \mathrm{a}$ & $118.33 \mathrm{a}$ & $136.00 \mathrm{a}$ & $65.93 \mathrm{a}$ & $36.83 \mathrm{a}$ & $31.33 \mathrm{a}$ & $1.85 \mathrm{~g}$ & $0.32 \mathrm{e}$ & $13.65 \mathrm{~d}$ & $12.32 \mathrm{c}$ \\
\hline 8 & $215.42 \mathrm{a}$ & $157.67 \mathrm{a}$ & $94.17 \mathrm{a}$ & $11.17 \mathrm{a}$ & $121.33 \mathrm{a}$ & $144.33 \mathrm{a}$ & $66.60 \mathrm{a}$ & $38.73 \mathrm{a}$ & $15.67 \mathrm{~b}$ & $2.23 \mathrm{f}$ & $0.34 \mathrm{e}$ & $13.46 \mathrm{~d}$ & $9.23 \mathrm{c}$ \\
\hline
\end{tabular}

$\mathrm{CD}=$ canopy diameter $(\mathrm{mm}), \mathrm{PH}=$ plant height $(\mathrm{mm}), \mathrm{FBH}=$ first bifurcation height $(\mathrm{mm}), \mathrm{SD}=$ stem diameter $(\mathrm{mm})$, $\mathrm{DTF}=$ days to flowering counted from transplanting date, DTFr $=$ days to fruiting counted from transplanting date, $\mathrm{LL}=$ leaf length $(\mathrm{mm}), \mathrm{LW}=$ leaf width $(\mathrm{mm}), \mathrm{FN}=$ fruit number per plant, $\mathrm{FM}=$ fruit mass $(\mathrm{g}), \mathrm{DMC}=$ fruit dry matter content $(\mathrm{g}), \mathrm{FL}=$ fruit length $(\mathrm{mm})$ and $\mathrm{FD}=$ fruit diameter $(\mathrm{mm})$. Equal letters in column represent same grouping by Scott-Knott criterion at $1 \%$ probability. 
Table 3. Analysis of variance and quadratic components of general combining ability $\left(\phi^{2} \mathrm{~g}\right)$, specific combining ability $\left(\phi^{2} s\right)$ and reciprocal $\left(\phi^{2} r\right)$ for 13 characteristics in ornamental pepper in Sete Lagoas in 2018.

\begin{tabular}{|c|c|c|c|c|c|c|c|c|}
\hline SV & GCA & SCA & Reciprocals & Error & $\phi^{2} \mathrm{~g}$ & $\phi^{2} \mathbf{s}$ & $\phi^{2} \mathbf{r}$ & $\phi^{2} g / \phi^{2} s$ \\
\hline $\mathrm{DF}$ & 7 & 28 & 28 & 128 & - & - & - & 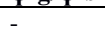 \\
\hline $\mathrm{CD}$ & $16039.40^{* *}$ & $1424.84^{* * *}$ & $2540.12^{* * *}$ & 434.08 & 325.11 & 330.25 & 351.01 & 0.98 \\
\hline $\mathrm{PH}$ & $6560.38^{* * *}$ & $757.68^{*}$ & $1118.75^{* *}$ & 421.57 & 127.89 & 112.04 & 116.20 & 1.14 \\
\hline FBH & $2382.25^{* *}$ & $441.13^{\mathrm{NS}}$ & $652.02^{* *}$ & 306.89 & 43.24 & 44.75 & 57.52 & 0.96 \\
\hline SD & $60.97^{* * *}$ & $3.94^{\mathrm{NS}}$ & $11.31^{\text {** }}$ & 3.05 & 1.21 & 0.30 & 1.38 & 4.03 \\
\hline DTF & $792.07^{* * *}$ & $140.69^{\mathrm{NS}}$ & $186.31^{\mathrm{NS}}$ & 133.77 & 13.71 & 2.31 & 8.76 & 5.94 \\
\hline DTFr & $952.84^{* *}$ & $182.21^{\mathrm{NS}}$ & $209.01^{\mathrm{NS}}$ & 158.08 & 16.56 & 8.04 & 8.49 & 2.06 \\
\hline LL & $3974.65^{* * *}$ & $98.77^{* *}$ & $964.82^{* * *}$ & 1.62 & 82.77 & 32.38 & 160.53 & 2.56 \\
\hline LW & $1039.88^{* * *}$ & $17.64^{* *}$ & $220.72^{* * *}$ & 1.53 & 21.63 & 5.37 & 36.53 & 4.03 \\
\hline FN & $347.17^{* *}$ & $24.79^{\mathrm{NS}}$ & $46.88^{* * *}$ & 23.18 & 6.75 & 0.54 & 3.95 & 12.50 \\
\hline FM & $3.95^{* *}$ & $0.74^{* *}$ & $0.92^{\text {** }}$ & 0.03 & 0.11 & 0.24 & 0.15 & 0.46 \\
\hline DMC & $0.35^{* *}$ & $0.02^{* * *}$ & $0.08^{* *}$ & 0.01 & 0.01 & 0.01 & 0.01 & 1.00 \\
\hline FL & $1153.51^{* *}$ & $64.63^{* *}$ & $352.55^{* *}$ & 10.74 & 23.81 & 17.96 & 56.97 & 1.33 \\
\hline FD & $152.05^{* *}$ & $11.46^{* *}$ & $52.22^{* *}$ & 3.11 & 3.10 & 2.78 & 8.18 & 1.12 \\
\hline \multicolumn{9}{|c|}{$\begin{array}{l}\mathrm{SV}=\text { sources of variation; } \mathrm{DF}=\text { degrees of freedom; GCA = general combining ability; SCA= specific combining } \\
\text { ability; } \mathrm{CD}=\text { canopy diameter }(\mathrm{mm}), \mathrm{PH}=\text { plant height }(\mathrm{mm}), \mathrm{FBH}=\text { first bifurcation height }(\mathrm{mm}), \mathrm{SD}=\mathrm{stem} \text { diameter } \\
(\mathrm{mm}), \mathrm{DTF}=\text { days to flowering counted from transplanting date, } \mathrm{DTFr}=\text { days to fruiting counted from transplanting } \\
\text { date, } \mathrm{LL}=\text { leaf length }(\mathrm{mm}), \mathrm{LW}=\text { leaf width }(\mathrm{mm}), \mathrm{FN}=\text { fruit number per plant, } \mathrm{FM}=\text { fruit mass }(\mathrm{g}), \mathrm{DMC}=\text { fruit dry } \\
\text { matter content }(\mathrm{g}), \mathrm{FL}=\text { fruit length }(\mathrm{mm}) \text { and } \mathrm{FD}=\text { fruit diameter }(\mathrm{mm}) . * * * \text { significant at } 1 \% \text { and at } 5 \% \text { probability } \\
\text { and NS: not significant by F-test. }\end{array}$} \\
\hline
\end{tabular}

The predominance of additive effects in the control of FBH, SD, DTF, DTFr and FN was also confirmed by the non-significance of effects of the specific combining ability (Table 3), evidencing the absence of dominance effects and other types of components of epistatic variance in their controls (Griffing, 1956). These results indicate the impossibility of exploring heterosis for these characteristics.

Nascimento et al. (2014), working with $C$. annuum, found that the additive effects were more important for fruit length and diameter and non-additive effects for days for fruiting. Silva et al. (2017), also working with $C$. annuum, verified that the additive effects were more important for plant height before and after fruiting, days for flowering, fruit length, fruit diameter and total number of fruits and non-additive effects for canopy diameter and days for fruiting.

Negative estimation of the effects of the general combining ability ( $\hat{\mathrm{g}}$ ) for plant height and for height of the first bifurcation were expressed by UFSJ 3 and UFSJ 5 parents (Table 4). When used in crosses, these genotypes will contribute to obtain plants of smaller size, desirable for ornamental purposes (Carvalho et al., 2006; Rêgo et al., 2015; Silva et al., 2015). Regarding canopy diameter, UFSJ 7 and UFSJ 8 expressed positive gi values. When used in crosses, these genotypes tend to produce visually more attractive hybrids due to the greater amount of foliage (Silva et al., 2017). UFSJ 7 and UFSJ 8 also expressed positive gi values for stem diameter and tend to produce hybrids with higher base of support for the plant. Also with regard to the good foliage density, UFSJ 4, UFSJ 7 and UFSJ 8 genotypes presented positive ĝ i values for leaf length and UFSJ 5, UFSJ 7 and UFSJ 8 genotypes presented positive gi values for leaf width (Table 4 ). 
Table 4. Estimates of general combining ability (ĝi) relative to 13 features in ornamental pepper at Sete Lagoas in 2018.

\begin{tabular}{|c|c|c|c|c|c|c|c|c|}
\hline Parents & 1 & 2 & 3 & 4 & 5 & 6 & 7 & 8 \\
\hline$\overline{C D}$ & $-11.65^{* *}$ & $-6.53 *$ & $-9.31 * *$ & -0.34 & $-20.17 * *$ & $-7.44^{*}$ & $21.60 * *$ & $33.84 * *$ \\
\hline PH & -0.44 & -4.75 & $-10.20 * *$ & -0.61 & $-14.85^{* *}$ & -3.54 & $15.44^{* * *}$ & $18.19 * *$ \\
\hline FBH & 0.76 & -1.71 & $-8.15^{* *}$ & 0.40 & $-9.09 * *$ & -1.80 & $9.93 * *$ & $9.64 * *$ \\
\hline SD & $-0.62 * *$ & $-1.18 * *$ & -0.06 & -0.32 & $-0.89 * *$ & -0.42 & $1.45^{* * *}$ & $1.99 * *$ \\
\hline DTF & -1.23 & 0.27 & -2.73 & $-3.06^{*}$ & -2.83 & -2.40 & $3.58 *$ & $8.40 * *$ \\
\hline DTFr & -1.84 & -0.16 & -2.18 & $-3.41^{*}$ & $-2.93 * *$ & -2.49 & $3.34 *$ & $9.66 * *$ \\
\hline LL & $-12.96^{* *}$ & $-1.16^{* *}$ & $-3.57 * *$ & $2.69 * *$ & $-3.85^{* *}$ & $-6.65^{* *}$ & $11.01 * *$ & $14.49 * *$ \\
\hline LW & $-6.58 * *$ & -0.32 & $-3.39 * *$ & $-1.37 * *$ & $1.31 * *$ & $-2.00 * *$ & $3.60 * *$ & $8.76^{* *} *$ \\
\hline FN & $-2.15 * *$ & -1.23 & $-2.15^{* *}$ & -0.89 & -1.26 & -0.67 & $1.62 *$ & $5.93 * *$ \\
\hline FM & $0.35^{* *}$ & $0.34 * *$ & $0.41 * *$ & $-0.31 * *$ & $-0.36^{* *}$ & $-0.26^{* *}$ & $0.07 * *$ & $-0.24 * *$ \\
\hline DMC & $0.07 * *$ & $0.12 * *$ & $0.12 * *$ & $-0.04 * *$ & $-0.09 * *$ & $-0.07 * *$ & $-0.03 * *$ & $-0.07 * *$ \\
\hline FL & $-4.26 * *$ & $10.74 * *$ & 0.21 & 0.15 & 0.72 & -0.73 & $-1.16^{* *}$ & $-5.68 * *$ \\
\hline FD & $-1.38 * *$ & $1.77 * *$ & 0.37 & $1.09 * *$ & $2.57 * *$ & -0.12 & $-2.17 * *$ & $-2.13 * *$ \\
\hline
\end{tabular}

In relation to precocity, negative gi values for DTFr were expressed by UFSJ 4 and UFSJ 5 genotypes. The UFSJ 4 genitor also presented precocity for variable DTF (Table 4). Silva et al. (2015) report the importance of precocity in ornamental peppers and consider that precocious plants are desirable to reduce production costs and promptly supply the market.

Regarding fruit length, diameter and mass, negative gi values are preferred in peppers for ornamental purposes since they favor the production of fruits of smaller dimensions but in greater number (Rêgo et al., 2010; Silva et al., 2015; Silva et al., 2017). Negative gi values for smaller fruit size were expressed by UFSJ 1, UFSJ 7 and UFSJ 8 parents and negative gi values for lower fruit mass were expressed by UFSJ 4, UFSJ 5, UFSJ 6 and UFSJ 8 parents. For number of fruits per plant, positive gi values were expressed by UFSJ 7 and UFSJ 8 parents (Table 4).

Regarding fruit dry matter content, according to Nascimento et al. (2015b), there is a direct relationship between increase of this content and greater abscission of ornamental pepper leaves due to the deleterious effects of ethylene. The favorable negative gi values for fruit dry matter content were expressed by UFSJ 4, UFSJ 5, UFSJ 6, UFSJ 7 and UFSJ 8 genotypes (Table 4).

The Sii parameter refers to the effect of the ability of a parent to combine with itself and is of fundamental importance to indicate the direction of the characteristic's dominance deviations. Sii will be negative when these deviations are predominantly positive. When Sii values are positive for some parents and negative for others, there is evidence of bidirectional dominance (Cruz and Vencovsky, 1989), thus by means of significant estimates of the Sii values of parents, unidirectional dominance was found for LW, FL and FD and bidirectional dominance for CD, LL and FM (Table 5). 
Table 5. Sii or Sij values and reciprocal effects (Rij) for 13 features in eight parents and 56 hybrids of ornamental pepper in Sete Lagoas in 2018.

\begin{tabular}{|c|c|c|c|c|c|c|c|c|c|c|c|}
\hline Accessions & CD & & & PH & & FBH & & SD & & DTF & \\
\hline 1 & -10.73 & & - & 14.27 & - & 5.56 & - & 1.28 & - & -3.77 & - \\
\hline $1 \times 2(2 \times 1)$ & 3.98 & & $(23.83) * *$ & 5.35 & $(21.17) *$ & 1.80 & $(18.14) *$ & 1.11 & $(1.28)$ & 4.23 & (3.17) \\
\hline $1 \times 3(3 \times 1)$ & 18.05 & * & (1.70) & 13.37 & $(6.92)$ & 15.51 & $(12.21)$ & 1.01 & $(0.39)$ & 0.23 & $(0.17)$ \\
\hline $1 \times 4(4 \times 1)$ & -5.21 & & $(25.99) * *$ & -9.63 & $(25.00) * *$ & -12.17 & $(15.74) *$ & -1.30 & $(0.13)$ & 1.17 & $(-3.17)$ \\
\hline $1 \times 5(5 \times 1)$ & 22.57 & $* *$ & $(-24.82) * *$ & 6.77 & $(2.84)$ & 8.40 & (1.16) & 0.63 & $(0.83)$ & 0.73 & $(-4.00)$ \\
\hline $1 \times 6(6 \times 1)$ & -16.89 & $*$ & $(-21.04) *$ & $-15.12 *$ & $(-2.75)$ & -13.05 & $(-13.17)$ & -0.44 & $(-1.08)$ & -0.92 & $(1.67)$ \\
\hline $1 \times 7(7 \times 1)$ & -6.76 & & $(-38.72) * *$ & $-15.84 *$ & $(-8.17)$ & -6.85 & $(-9.11)$ & -1.62 & $(-1.21)$ & 0.10 & $(-4.67)$ \\
\hline $1 \times 8(8 \times 1)$ & -5.01 & & $(-37.29) * *$ & 0.83 & $(-17.17) *$ & 0.80 & $(-7.14)$ & -0.68 & $(-2.02) * *$ & -1.44 & $(-10.17)$ \\
\hline 2 & -7.14 & & - & 1.67 & - & -6.47 & - & -0.31 & - & -10.44 & - \\
\hline $2 \times 3(3 \times 2)$ & 33.40 & $* *$ & $(-15.92)$ & 1.02 & $(-9.58)$ & 2.17 & $(-7.6)$ & 0.39 & $(0.37)$ & 1.06 & $(-0.33)$ \\
\hline $2 \times 4(4 \times 2)$ & 4.18 & & $(-20.17) *$ & 14.20 & $(-8.73)$ & 10.73 & $(-5.33)$ & 0.69 & $(-0.81)$ & 3.33 & (4.17) \\
\hline $2 \times 5(5 \times 2)$ & -0.64 & & (4.65) & 5.60 & (15.67) & 0.61 & $(18.57) * *$ & -0.27 & $(0.66)$ & 1.40 & $(2.67)$ \\
\hline $2 \times 6(6 \times 2)$ & -11.24 & & $(-1.35)$ & -3.70 & $(-5.82)$ & -1.43 & $(-0.35)$ & -0.59 & $(0.43)$ & 1.40 & $(9.16)$ \\
\hline $2 \times 7(7 \times 2)$ & 0.38 & & $(-12.61)$ & -4.80 & $(-12.83)$ & -1.64 & $(-8.67)$ & -0.63 & $(-1.38)$ & 4.58 & $(-2.00)$ \\
\hline $2 \times 8(8 \times 2)$ & -22.92 & ** & $(-29.08) * *$ & $-19.33 * *$ & $(-23.64) * *$ & -5.77 & $(-16.02) *$ & -0.41 & $(-2.77) * *$ & 5.27 & $(-6.17)$ \\
\hline 3 & -24.74 & * & - & -13.13 & - & -10.29 & - & -1.77 & - & -2.44 & - \\
\hline $3 \times 4(4 \times 3)$ & 10.25 & & $(0.82)$ & -9.06 & (1.68) & -2.89 & $(2.55)$ & 0.16 & $(-0.33)$ & -1.77 & $(-7.67)$ \\
\hline $3 \times 5(5 \times 3)$ & -27.46 & $* *$ & (12.58) & $-15.39 *$ & $(1.92)$ & -17.18 & $(-0.83)$ & 0.59 & (1.19) & 2.5 & (1.83) \\
\hline $3 \times 6(6 \times 3)$ & -23.17 & $* *$ & $(23.77) * *$ & -11.35 & (5.93) & -8.16 & (10.15) & -0.50 & $(2.02) * *$ & -3.27 & $(-5.17)$ \\
\hline $3 \times 7(7 \times 3)$ & -5.76 & & $(-20.38) *$ & 10.36 & $(-5.71)$ & 7.75 & $(-10.29)$ & 0.22 & $(-2.16) * *$ & 3.42 & $(-7.17)$ \\
\hline $3 \times 8(8 \times 3)$ & 19.43 & $* *$ & $(-17.18) *$ & $24.19 * *$ & $(-17.56) *$ & 13.09 & $(-1.67)$ & -0.10 & $(-1.38)$ & 7.77 & $(-3.00)$ \\
\hline 4 & 8.50 & & - & 15.37 & - & 9.62 & - & 0.54 & - & 0.56 & - \\
\hline $4 \times 5(5 \times 4)$ & -3.58 & & $(22.93) * *$ & -6.83 & $(16.39) *$ & -5.40 & (12.00) & 0.33 & $(0.22)$ & 1.5 & $(-5.83)$ \\
\hline $4 \times 6(6 \times 4)$ & -6.75 & & (11.73) & 4.45 & $(-1.65)$ & 2.73 & $(0.75)$ & -0.26 & $(0.93)$ & -1.60 & $(-9.83)$ \\
\hline $4 \times 7(7 \times 4)$ & -4.61 & & $(-22.67) * *$ & -1.00 & $(-4.82)$ & 1.69 & $(-10.25)$ & -0.26 & $(-0.28)$ & 0.42 & $(-7.5)$ \\
\hline $4 \times 8(8 \times 4)$ & -2.78 & & $(-31.73) * *$ & -7.50 & $(-18.5) *$ & -4.29 & $(-8.5)$ & 0.10 & $(-3.45) * *$ & -0.40 & $(-10.5)$ \\
\hline 5 & 6.84 & & - & 4.65 & - & 6.30 & - & 0.05 & - & -10.23 & - \\
\hline $5 \times 6(6 \times 5)$ & -1.51 & & $(-15.73)$ & -0.21 & $(-10.65)$ & 4.75 & $(-19.82) * *$ & -0.00 & $(0.78)$ & 9.00 & (1.33) \\
\hline $5 \times 7(7 \times 5)$ & 5.30 & & (6.75) & 8.56 & (10.83) & -1.10 & (4.17) & -0.02 & $(-0.30)$ & 3.52 & $(-1.83)$ \\
\hline $5 \times 8(8 \times 5)$ & -1.54 & & $(-10.85)$ & -3.15 & $(5.64)$ & 3.62 & $(8.58)$ & -1.30 & $(-1.13)$ & -10.80 & $(1.33)$ \\
\hline 6 & 23.28 & * & - & 7.40 & - & -2.17 & - & -0.17 & - & 0.90 & - \\
\hline $6 \times 7(7 \times 6)$ & 25.99 & $* *$ & $(0.83)$ & $15.83 *$ & $(0.42)$ & 15.36 & $(3.25)$ & 0.84 & $(-0.70)$ & -1.75 & $(-6.00)$ \\
\hline $6 \times 8(8 \times 6)$ & 10.30 & & $(-30.62) * *$ & 2.69 & $(-29.83) * *$ & 1.99 & $(-12.25)$ & 1.12 & $(-2.40) * *$ & -5.40 & $(-6.83)$ \\
\hline 7 & -19.02 & & - & -14.90 & - & -13.62 & - & 0.06 & - & -9.73 & - \\
\hline $7 \times 8(8 \times 7)$ & 4.8 & & $(-1.78)$ & 1.79 & $(-19.5) *$ & -1.57 & $(-7.75)$ & 1.42 & $(-0.32)$ & 0.46 & $(-4.33)$ \\
\hline 8 & -1.95 & & - & 0.47 & - & -7.86 & - & -0.14 & - & 2.98 & - \\
\hline Accessions & DTFr & & & $\mathbf{L L}$ & & LW & & FN & & FM & \\
\hline 1 & -1.66 & & - & 0.06 & - & 0.13 & - & 0.13 & - & $1.06 * *$ & - \\
\hline $1 \times 2(2 \times 1)$ & -5.84 & & (3.17) & 0.76 & $(-12.37) * *$ & 0.29 & $(-6.00) * *$ & -0.95 & $(1.50)$ & $-0.53 * *$ & $(0.30) * *$ \\
\hline $1 \times 3(3 \times 1)$ & 5.51 & & $(-2.17)$ & -0.60 & $(-8.73) * *$ & 0.54 & $(-2.82) * *$ & 0.96 & $(1.50)$ & $-0.30 * *$ & $(0.11)$ \\
\hline $1 \times 4(4 \times 1)$ & 2.07 & & $(-4.5)$ & $1.29 * *$ & $(-16.05) * *$ & -0.21 & $(-4.62) * *$ & -2.60 & $(2.00)$ & $0.22 * *$ & $(-0.56) * *$ \\
\hline $1 \times 5(5 \times 1)$ & 0.59 & & $(-4.17)$ & $1.12 *$ & $(-9.82) * *$ & 0.12 & $(-8.14) * *$ & 0.57 & $(0.33)$ & 0.03 & $(0.17) *$ \\
\hline $1 \times 6(6 \times 1)$ & -0.34 & & $(3.00)$ & $-2.14 * *$ & $(-3.32) * *$ & -0.44 & $(-4.00) * *$ & 0.65 & $(2.00)$ & $-0.21 * *$ & $(-0.20) * *$ \\
\hline $1 \times 7(7 \times 1)$ & -1.84 & & $(-6.67)$ & 0.65 & $(-23.77) * *$ & 0.32 & $(-10.33) * *$ & -0.81 & $(-1.17)$ & 0.05 & $(0.79) * *$ \\
\hline $1 \times 8(8 \times 1)$ & 1.51 & & $(-10.33)$ & $-1.15 *$ & $(-25.62) * *$ & -0.75 & $(-14.22) * *$ & 2.05 & $(-1.00)$ & $-0.34 * *$ & $(0.44) * *$ \\
\hline 2 & -2.03 & & - & $1.47 *$ & - & 0.48 & - & -3.70 & - & $-0.98 * *$ & - \\
\hline $2 \times 3(3 \times 2)$ & -10.17 & & $(0.50)$ & 0.41 & $(3.15) * *$ & 0.63 & $(3.18) * *$ & 0.55 & (2.33) & $0.32 * *$ & $(0.36) * *$ \\
\hline $2 \times 4(4 \times 2)$ & 3.05 & & (2.17) & $1.72 * *$ & $(-3.45) * *$ & -0.16 & $(1.87) * *$ & 0.65 & $(-1.83)$ & $0.71 * *$ & $(-0.53) * *$ \\
\hline $2 \times 5(5 \times 2)$ & 2.24 & & (1.17) & $1.27 * *$ & $(2.73) * *$ & $1.63 * *$ & $(-1.36) * *$ & 0.48 & $(2.50)$ & $0.15 *$ & $(0.29) * *$ \\
\hline $2 \times 6(6 \times 2)$ & 1.47 & & $(5.50)$ & $-1.74 * *$ & $(9.55) * *$ & 0.49 & $(2.97) * *$ & 1.57 & $(-2.50)$ & $0.14 *$ & $(0.37) * *$ \\
\hline $2 \times 7(7 \times 2)$ & 6.47 & & $(-4.67)$ & $1.00 *$ & $(-11.82) * *$ & 0.61 & $(-3.26) * *$ & 1.61 & (1.17) & 0.00 & $(-0.01)$ \\
\hline $2 \times 8(8 \times 2)$ & 4.82 & & $(-6.00)$ & $-4.90 * *$ & $(-17.27) * *$ & $-3.97 * *$ & $(-11.30) * *$ & -0.20 & $(-1.67)$ & $0.19 * *$ & $(0.86) * *$ \\
\hline 3 & -3.32 & & - & -0.86 & - & -0.34 & - & -3.54 & - & $0.26 * *$ & - \\
\hline $3 \times 4(4 \times 3)$ & -2.43 & & $(-5.67)$ & 0.17 & $(-7.15) * *$ & -0.71 & $(-1.52) * *$ & -1.10 & (1.17) & $-0.25 * *$ & $(0.36) * *$ \\
\hline $3 \times 5(5 \times 3)$ & 1.93 & & (5.17) & 0.53 & $(-1.52) * *$ & 0.66 & $(-5.13) * *$ & -1.77 & (1.67) & $0.21 * *$ & $(0.27) * *$ \\
\hline $3 \times 6(6 \times 3)$ & -2.84 & & $(-2.50)$ & $-3.38 * *$ & $(4.80) * *$ & -0.20 & $(-1.00) *$ & 0.82 & $(4.17) \quad *$ & 0.11 & $(0.50) * *$ \\
\hline $3 \times 7(7 \times 3)$ & 3.99 & & $(-10.17)$ & $-1.57 * *$ & $(-14.93) * *$ & -0.36 & $(-6.40) * *$ & 3.19 & $(-2.50)$ & $-0.23 * *$ & $(0.64) * *$ \\
\hline $3 \times 8(8 \times 3)$ & 7.34 & & $(-3.50)$ & $5.31 * *$ & $(-10.20) * *$ & -0.21 & $(-9.02) * *$ & 0.88 & $(-4.17) *$ & $-0.13 *$ & $(0.44) * *$ \\
\hline 4 & -1.86 & & - & $2.19 * *$ & - & 0.35 & - & 1.67 & - & 0.09 & - \\
\hline $4 \times 5(5 \times 4)$ & 4.66 & & $(-5.67)$ & $1.87 * *$ & $(6.42) * *$ & 0.51 & $(-3.13) * *$ & 3.84 & $(0.33)$ & $-0.54 * *$ & $(0.01)$ \\
\hline $4 \times 6(6 \times 4)$ & -1.95 & & $(-9.50)$ & $-1.07 *$ & $(12.87) * *$ & -0.70 & $(0.75)$ & 1.59 & $(-1.67)$ & -0.04 & $(-0.11)$ \\
\hline $4 \times 7(7 \times 4)$ & -0.95 & & $(-7.33)$ & -0.20 & $(-7.37) * *$ & $-1.33 * *$ & $(-4.58) * *$ & -1.37 & (1.00) & $-0.25 * *$ & $(-0.39) * *$ \\
\hline $4 \times 8(8 \times 4)$ & -2.60 & & $(-12.33)$ & $-5.98 * *$ & $(-17.37) * *$ & $2.25 * *$ & $(-6.53) * *$ & -2.68 & $(-5.33) * *$ & 0.06 & $(0.13)$ \\
\hline 5 & -8.16 & & - & $3.06 * *$ & - & $1.53 *$ & - & -0.66 & - & $-0.43 * *$ & - \\
\hline $5 \times 6(6 \times 5)$ & 9.58 & & $(1.50)$ & $-1.40 * *$ & $(6.37) * *$ & -0.00 & $(4.27) * *$ & -1.74 & (1.17) & -0.09 & $(-0.10)$ \\
\hline $5 \times 7(7 \times 5)$ & 2.41 & & $(-3.50)$ & $1.07 *$ & $(-13.60) * *$ & 0.14 & $(-1.53) * *$ & -3.04 & $(-0.17)$ & $0.28 * *$ & $(-0.47) * *$ \\
\hline $5 \times 8(8 \times 5)$ & -13.24 & & $(-1.17)$ & $-7.51 * *$ & $(-23.50) * *$ & $-4.58 * *$ & $(-11.25) * *$ & 2.32 & $(-0.17)$ & $0.38 * *$ & $(0.14) *$ \\
\hline 6 & 1.30 & & - & $-4.43 * *$ & - & -0.53 & - & 2.17 & - & -0.07 & - \\
\hline $6 \times 7(7 \times 6)$ & 0.14 & & $(-6.33)$ & $-1.71 * *$ & $(-19.38) * *$ & $-2.10 * *$ & $(-5.02) * *$ & -1.12 & (2.33) & $0.32 * *$ & $(-0.26) * *$ \\
\hline $6 \times 8(8 \times 6)$ & -7.34 & & $(-7.83)$ & $15.87 * *$ & $(-3.05) * *$ & $3.48 * *$ & $(-3.60) * *$ & -3.93 & $(-8.83) * *$ & $-0.16 * *$ & $(-0.13)$ \\
\hline 7 & -10.70 & & - & 0.37 & - & -0.94 & - & 1.26 & - & 0.09 & - \\
\hline $7 \times 8(8 \times 7)$ & 0.49 & & $(-6.50)$ & 0.38 & $(-0.42)$ & $3.65 * *$ & $(0.23)$ & 0.28 & $(-4.00) *$ & $-0.26 * *$ & $(0.18) *$ \\
\hline 8 & 9.01 & & - & $-2.02 * *$ & - & 0.12 & - & 1.30 & - & $0.26 * *$ & - \\
\hline
\end{tabular}




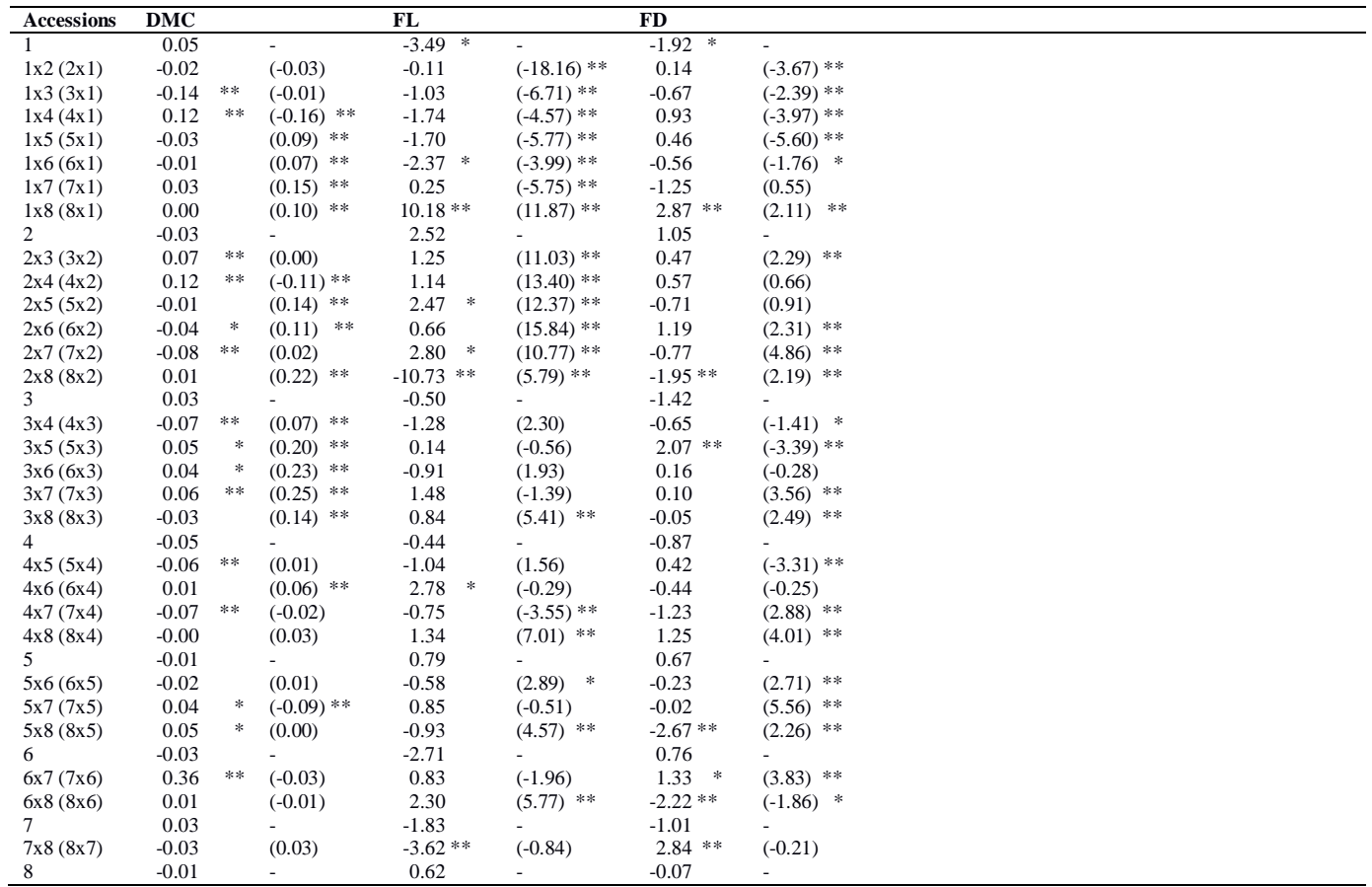

$\mathrm{CD}=$ canopy diameter $(\mathrm{mm}), \mathrm{PH}=$ plant height $(\mathrm{mm}), \mathrm{FBH}=$ first bifurcation height $(\mathrm{mm}), \mathrm{SD}=$ stem diameter $(\mathrm{mm})$, $\mathrm{DTF}=$ days to flowering counted from transplanting date, DTFr $=$ days to fruiting counted from transplanting date, $\mathrm{LL}=$ leaf length $(\mathrm{mm}), \mathrm{LW}=$ leaf width $(\mathrm{mm}), \mathrm{FN}=$ fruit number per plant, FM = fruit mass $(\mathrm{g}), \mathrm{DMC}=$ fruit dry matter content $(\mathrm{g}), \mathrm{FL}=$ fruit length $(\mathrm{mm})$ and $\mathrm{FD}=$ fruit diameter $(\mathrm{mm}) . * * *$ significant at $1 \%$ and at $5 \%$ probability by t-test.

Sii estimates were in favor of selection for increased CD in UFSJ 3 parent, for increased LL in UFSJ 6 and UFSJ 8 parents, and decreased MF in UFSJ 1, UFSJ 3 and UFSJ 8 parents (Table 5). Although parental UFSJ 8 presented such favorable Sii estimates, all its hybrids inherited the pending position of fruits, which is a limiting factor in the selection of ornamental peppers.

Reciprocal effects were highly significant for all traits, except for DTF and DTFr (Table 3). Bordallo et al. (2005) and Mahgoub (2011) reported that even when the effects of specific combining ability are not significant, heterosis can be explored based on the means of squares of reciprocal effects, since there is a parent contributing with their cytoplasmic inheritance and/or with their maternal effect in determining the characteristic. In this way, hybrid combinations with greater possibilities of presenting such effects and with at least one parent with favorable gi effect (Cruz and Regazzi, 1994) were selected.

Based on the good classification of UFSJ 1, UFSJ 3 and UFSJ 6 parents in relation to Sii values (Table 5), considering the significant and favorable reciprocal effects for at least three characteristics simultaneously (Table 5) and the existence of at least one of parents with gi values in favor of the selection (Table 4), UFSJ 4 X UFSJ 1 and UFSJ 6 X UFSJ 1 hybrids were selected. No crosses involving the previously selected UFSJ 3 parent based on the Sii value satisfied the other two selection requirements. The UFSJ 4 X UFSJ 1 crossing showed favorable and significant reciprocal effects on the increase of CD and reduction of FM, DMC, LL and FD (Table 5) and presented at least one parent with favorable gi values for FM, DMC, FL and FD (Table 4). The UFSJ 6 X UFSJ 1 crossing 
had favorable and significant reciprocal effects on the reduction of FM, FL and FD (Table 5) and presented at least one parent with favorable gi values for these three characteristics (Table 4). These hybrids are the result of good genetic complementation among their parents and indicate the possibility of obtaining genetic gains through the exploration of heterosis in future generations of ornamental peppers by means of the development of lineages (Reddy and Joshi, 1993).

\section{CONCLUSIONS}

Most of the characteristics evaluated in ornamental peppers, except for fruit mass, can be improved by simple selection methods due to the predominantly additive genetic control. UFSJ 4 x UFSJ 1 and UFSJ 6 x UFSJ 1 hybrid combinations were recommended to obtain improved lines.

\section{CONFLICTS OF INTEREST}

The authors declare no conflict of interest.

\section{REFERENCES}

Ben-Chaim A and Paran I (2000). Genetic analysis of quantitative traits in pepper (Capsicum annuum). J. Am. Soc. Hortic. Sci. 125: 66-70.

Bordallo PN, Pereira MG, Amaral Júnior AT and Gabriel APC (2005). Análise dialélica de genótipos de milho doce e comum para caracteres agronômicos e proteína total. Hortic. Bras. 23: 123-127.

Carvalho SIC, Bianchetti LB, Ribeiro CSC and Lopes CA (2006). Pimentas do gênero Capsicum no Brasil. Embrapa Hortaliças, Brasília.

Cruz CD (2006). Programa genes: aplicativo computacional em genética e estatística. UFV, Viçosa.

Cruz CD and Regazzi AJ (1994). Modelos biométricos aplicados ao melhoramento genético. UFV, Viçosa.

Cruz CD and Vencovsky R (1989). Comparação de alguns métodos de análise dialélica. Rev. Bras. Genet. 12: 425-438.

Doshi KM and Shukla PT (2000). Combining ability analysis for fresh fruit yield and its components over environments in chili (Capsicum annuum). Capsicum Eggplant Newsl. 19: 82-85.

Gonçalves LSA, Rodrigues R, Bento CS, Robaina RR, et al. (2011). Herança de caracteres relacionados à produção de frutos em Capsicum baccatum var. pendulum com base em análise dialélica de Hayman. Cienc. Agron. 42: 662669.

Griffing B (1956). Concept of general and specific combining ability in relation to diallel crossing systems. Aust. J. Biol. Sci. 9: 463-493.

Heiden G, Barbieri RL, Stumpf ERT (2006). Considerações sobre o uso de plantas ornamentais nativas. Rev. Bras. Hortic. Ornam. 12: 2-7.

International Plant Genetic Resources Institute (1995). Descriptors for Capsicum. IBPGR, Rome.

Kamble C, Mulge RM and Madalageri B (2009). Combining ability for earliness and productivity in sweet pepper (Capsicum annuum L.). Karnataka J. Agric. Sci. 22: 151-154.

Mahgoub GMA (2011). Partitioning of general and specific combining ability effects for estimating maternal and reciprocal effects. J. Agric. Sci. 3: 213-221.

Medeiros AM, Rodrigues R, Gonçalves LSA, Sudré CP, et al. (2014). Gene effect and heterosis in Capsicum baccatum var. pendulum. Cienc. Rural. 44: 1031-1036.

Nascimento MF, Nascimento NFF, Rêgo ER, Bruckner CH, et al. (2015a). Genetic diversity in a structured family of six generations of ornamental chili peppers (Capsicum annuum). Acta Hortic. 1087: 395-401.

Nascimento NFF, Rêgo ER, Nascimento MF, Bruckner CH, et al. (2014). Combining ability for yield and fruit quality in the pepper Capsicum annuum. Genet. Mol. Res. 13: 3237-3249.

Nascimento MF, Rêgo ER, Nascimento NFF, Santos RMC, et al. (2015b). Correlation between morphoagronomic traits and resistance to ethylene action in ornamental peppers. Hortic. Bras. 33: 151-154.

Ramalho MAP, Santos JB and Pinto CABP (2008). Genética na Agropecuária. UFLA, Lavras.

Reddy JN and Joshi P (1993). Heterosis, inbreeding depressions and combining ability in sorghum (Sorghum bicolor (L.) Moench). Indian J. Genet. 53: 138-146. 
Reddy MG, Kumar HDM and Salimath PM (2008). Combining ability analysis in chilli (Capsicum annuum L.). Karnataka J. Agric. Sci. 21: 494-497.

Rêgo ER, Rêgo MM, Cruz CD, Finger FL, et al. (2010). Phenotypic diversity, correlation and importance of variables for fruit quality and yield traits in Brazilian peppers (Capsicum baccatum). Genet. Resour. Crop Evol. 58: 909-918.

Rêgo ER, Rêgo MM and Finger FL (2015). Methodological basis and advances for ornamental pepper breeding program in Brazil. Acta Hortic. 1087: 309-314.

Rêgo ER, Rêgo MM, Finger FL, Cruz CD, et al. (2009). A diallel study of yield components and fruit quality in chilli pepper (Capsicum baccatum). Euphytica. 168: 275-287.

Reifschneider FJB (2000). Capsicum: pimentas e pimentões no Brasil. Embrapa Hortaliças, Brasília.

Rodrigues R, Gonçalves LSA, Bento CS, Sudré CP, et al. (2012). Combining ability and heterosis for agronomic traits in chili pepper. Hortic. Bras. 30: 226-233.

Santos RMC, Rêgo ER, Borém A, Nascimento MF, et al. (2014). Epistasis and inheritance of plant habit and fruit quality traits in ornamental pepper (Capsicum annuum L.). Genet. Mol. Res. 13: 8876-8887.

Schuelter AR, Pereira GM, Amaral Júnior AT, Casali VWD, et al. (2010). Genetic control of agronomically important traits of pepper fruits analyzed by Hayman's partial diallel cross scheme. Genet. Mol. Res. 9: 113-127.

Silva CQ, Jasmim JM, Santos JO, Bento CS, et al. (2015). Phenotyping and selecting parents for ornamental purposes in chili pepper accessions. Hortic. Bras. 33: 66-73.

Silva CQ, Rodrigues R, Bento CS and Pimenta S (2017). Heterosis and combining ability for ornamental chili pepper. Hortic. Bras. 35: 349-357. 